\title{
Perspective Models As a Means For Achieving True Representational Accuracy
}

\author{
Kym Pohl \\ CDM Technologies Inc. \\ 2975 McMillan Ave. \\ San Luis Obispo, CA. 93401 \\ 805-541-3750 x233 \\ kpohl@cdmtech.com
}

\begin{abstract}
Accurate and expressive representation of the subject matter over which a context-oriented, decisionsupport system operates is fundamental to the effectiveness and longevity of the resulting solution. Often taking the form of an ontology, such extensive representational models, by their very nature, are rich in both relationships and fine-grained objects. It is, however, these two strengths that can significantly increase complexity for its users in addition to adversely affecting system performance. Further, due to the multitude of compartmentalized facets (i.e., populations of distinct, reasoning agents) inherent in such software solutions, it is important to recognize that a singleminded omniscient set of domain descriptions representing a singular view of the world is not necessarily appropriate for every ontology user. In fact, in such highly expressive environments, it is critical to not only accept these distinctions in user perspective, but to, in fact, promote and exploit them. It is by acknowledging and supporting this perspective-based individuality that true representational accuracy and utility is achieved.
\end{abstract}

Traditionally, software-based users comprising decisionsupport systems have operated over a singular, common representation, albeit a potential subset of the entire target modeling space. However, in the perspective modelenriched environment presented in this paper, ontology users are empowered with the ability to effectively perceive the world in accordance with individualized views. Architecturally, perspective models are integrated with one another via a central ontology. In this sense, perspective models act as satellites deriving certain aspects of their content from a central integration model. Exclusively operating over personalized perspective models, users are not only shielded from the broad-scoped complexities inherent in the more omniscient concerns of the central integration model but are also able to view and interact with the world in terms of their more familiar and expressive native representation.

To be effective, the concept of perspective models must be partnered with a supportive model development process. In addition to an explanation of the concept of perspective models, this paper provides a discussion of a development process that supports effective development of both the potentially numerous set of perspective models in addition to the integration model that inter-connects them. The process offered in this paper effectively parcels the development of individual perspective models with the individuals possessing the necessary domain and use-case expertise. In this manner, the development process strives to significantly increase the involvement of the entire set of team members in the modeling activity, both capitalizing on user domain expertise in addition to increasing critical user understanding and acceptance of the representation over which their components are to operate.

\section{TABle OF CONTENTS}

1. REPRESENTING PERSPECTIVE......................................1

2. AN EFFECTIVE DEVELOPMENT PROCESS ......................5

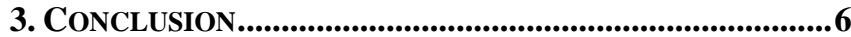

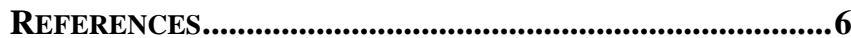

AUTHOR BIOGRAPHY .......................................................7

\section{RePresenting Perspective}

Fundamental to context-oriented reasoning is the highly expressive representation over which intricate analysis is performed [8] [12] [13]. Often taking the form of an ontology, such elaborate subject matter descriptions form the foundation critical to the effectiveness of contextoriented, decision-support systems. An ontology in the scope of this paper ${ }^{1,2}$ is defined as a highly expressive, typically relationship-rich model of the extensive subject matter over which software components, hereunto referred to as users, reason and otherwise operate.

\section{The Significance of Perspective}

Perspective is applied each time we as human beings perceive a particular subject matter. Housed within these individualized perspectives is valuable information describing how a particular topic is most suitably represented from a certain point of view. In addition, such perspectives also convey how that particular subject relates to other subject matter seen as relevant by the individual.

\footnotetext{
${ }^{1}$ 0-7803-9546-8/06/\$20.00@ 2006 IEEE

2 IEEEAC paper \#1381, Version 3, Updated November 30, 2005
} 
Even when a subject is shared among multiple individuals, perception is, more often than not, biased in favor of an individual's personal experiences and knowledge. Although at times a significant complication for meaningful interaction, such perspective is extremely significant to accurate representation as it is rich in descriptive context. For example, consider the following illustration involving the laptop on which this paper was written. In the case of a software system assisting the manufacturing process, the laptop might be most effectively described in terms of its product-oriented nature. In this sense, the most suitable representation of the laptop would revolve around characteristics dealing with assembly, packaging, and other aspects relevant to the manufacturing process. Further, relationships to customer orders and delivery schedules would also be important to represent. In contrast, however, characteristics explicitly describing the laptop's utility in authoring conference papers or developing software are fairly peripheral, if not completely irrelevant, to the manufacturing process. However, such perspective may be quite relevant to a system supporting, for example, the activities of marketing or perhaps customer-support. Both perspectives are quite valid with respect to their individual areas of operation. However, both views would encompass some of the same set of subjects (i.e., laptops) yet describe them in distinctively different manners. The problem arises when users of distinctly different representations of the same subject matter are required to interoperate. This situation can produce a significant dilemma. Simply stated, the valuable context that is expressed within individualized perspectives can also significantly limit their user's ability to interoperate with other users in a meaningful fashion (i.e., in terms of rich context).

However, despite these complications provoked by attempts to capture and exploit distinctive perceptions of relevant domains such descriptive expression is the ingredient in a context-oriented, decision-support environment that achieves true representation of context at the individual level. Perspective-enriched models can successfully capture not only the sometimes subtle distinctions among ontology users, but by doing so can promote a more expressive description of each user's perception of their world. Unfortunately, due to the complexity inherent in identifying and supporting such subtleties and nuances, representation approaching this level of expression has traditionally been buried as implied assumptions within convoluted business logic or simply omitted entirely. However, when appropriately represented and housed within the context tier of a collaborative environment, such expressiveness can not only be effectively exploited, but is also readily accessible to other users exhibiting similar views.

\section{Perspective Models}

However, even with expressive perspective sufficiently represented within the context tier, the ability for perspective users to interact based on these individualized views of the world poses a substantially complex interoperability problem. The solution to this interoperability dilemma primarily takes three forms. The first focuses on the development of a singular, omniscient ontology. Such an ontology would represent a monolithic, all-inclusive description of the world. Individual perspectives would only indirectly influence such model development in favor of describing subject matter as a complex concoction essentially merging both innate and biased characteristics with little delineation between the two. Each system would utilize this description as its sole representational means for operation and interaction with other users. In a positive sense, each user would essentially dialogue with one another in terms of a singular representation promoting interoperability in a clear and concise manner void of any context-diminishing translation. Each user would share the same exact, pseudo-biased view (i.e., a view based on an amalgamation of all relevant perspectives). However, considering the complexity resulting from collapsing what could possibly be numerous perspective-oriented characteristics into a single description of a particular subject matter, the resulting model would be severely bloated and would most likely fail to adequately represent any one particular perspective, resulting in a model confusing to utilize.

The second, somewhat related approach to this dilemma addresses the inevitable complexity of a singular, omniscient ontology offering a more delineated organization. In this approach, each particular subject matter is modeled in terms of its fundamental, intrinsic nature. The various perspectives applied to each particular subject are each explicitly represented as individual model fragments. These perspective sub models are connected to the subject models they enhance using the role analysis pattern [3]. Such a connection can be conceptualized as a subject potentially playing a variety of roles with each role representing a particular view on that subject. In this fashion, individual perspectives can be easily managed and clearly discernable from one another. In addition, this approach offers a degree, although limited, of encapsulation and isolation from irrelevant perspectives as users can isolate their interaction with a subject matter to those perspectives that are meaningful to them. Further, additional perspectives can be integrated in a manageable fashion through the incorporation of new roles-based model fragments. As a result, each subject is related to model fragments describing the various contexts in which it can be viewed. For example, interaction with the aforementioned laptop in terms of its manufacturing-oriented properties may be in terms of a related ManufacturedProductRole model fragment. However, the problem with this approach is that even though perspectives relating to the same subject matter are somewhat partitioned from one another, they remain integrated into a single model limiting true perspective isolation to diligent usage with no explicit management. As such, additional access control may need to be employed to truly isolate users to relevant perspectives. In addition, there is still the dilemma of whether or not a slight difference in two perspectives is worthy to warrant creation of an entirely 
new perspective model fragment. In practice, one would be tempted to collapse subtle differences in perspective into a single, overloaded model fragment, thus compromising accurate expression. Although these shortcoming are of a subtle nature, they are nonetheless noteworthy concerns.

The third, more promising solution to supporting multiple, isolated perspectives on a single subject matter introduces the notion of a perspective model. Based on a semi-stateful façade design pattern [5], perspective models allow contextrich subject matter to be viewed by inter-operating users in terms of individualized, native perspective. Perspective models may either directly contain their content or derive it from some type of shared source. While state simply for local consumption is represented and maintained within the perspective model itself, derivation is used for material that is shared across users (i.e., the basis for collaboration). In the case of derived content, the function of the perspective model may, for example, be to apply more native terminology, structure, or other characteristics that more appropriately represent the expressive perspective exhibited by the particular user. In some cases such mappings, either uni- or bi-directional, may be fairly straightforward and easily describable through standard expression grammar. However, in other cases these mappings may be rather complex to the point of requiring customized behavior. In either case, such mappings can be effectively described in terms of a formalized language such as XSLT [1] [9] or CLIPS-based rule sets [6] [11].

\section{Integration Model}

The derived nature of a perspective model is essentially the means for linking together multiple perspectives applied to the same subject matter. While there are a number of approaches to supporting such integration, it is critical that the individuality and bias exhibited by each perspective model is preserved in its native form. These models are essentially a user's most familiar and descriptive language with which to interact with the rest of the world (i.e., other users) and should not be corrupted in favor of more straightforward interaction with other parts of the environment.

The approach presented in this paper to interconnecting disparate perspectives of the same subject matter employs the notion of an integration model in conjunction with the façade design pattern [5]. Architecturally, this approach takes the form of a series of satellite perspective models interconnected via a central integration model. With this approach, a central, role-based representation of clearly delineated perspectives, not unlike the second alternative to integrating multiple perspectives described earlier, is developed as a well-structured and delineated combination of individualized perspectives related to the intrinsic subject matter they enhance. For example, the main subject of our earlier example might take the form of a laptop entity that can play the role of a manufactured product, as well as the role of a software platform. While the laptop entity would describe the subject's intrinsic nature, characteristics specific to each of these two perspectives would be housed within each related role.

As a further, diagrammatic description of this connection, Figure 1 describes a logistically-oriented perspective model linked to an integration model that presents a fairly neutral description of a conveyance. As an aside, note that conceptually such neutrality is not necessarily a prerequisite in that if the integration model were more heavily biased toward a particular perspective, it would simply imply that the perspective models might need to be more extensive and incorporate additional constraints. However, in the interest of clarity, this example employs a somewhat neutral integration model.

Central to the logistics perspective presented in Figure 1 is the notion of a transport. Although the logistics perspective may have knowledge of the entire set of conveyance types (i.e., vessels, vehicles, and aircraft) represented in the integration model, in respect to the logistics view, only vessels and rotary aircraft are considered candidate transports. In this situation, it would be valuable to represent this refined constraint in the perspective model employed by the logistics system while still basing such a biased view on the much more neutral representation of the conveyance offered by the integration model. As Figure 1 illustrates, representing such refinement can be accomplished by explicitly introducing a constrained notion of a transport in the logistics-oriented perspective model. According to the particular perspective, an abstract Transport is defined take two specific forms (VesselTransport and HelicopterTransport). At this point, it is immediately apparent that a vehicle is not a candidate to be a transport. In the context of this example, transports can only be VesselTransports or HelicopterTransports. The task now becomes linking this perspective together with the core integration model. Relating these two transport types to their conveyance derivation can be achieved in either an explicit or implicit manner. For illustration purposes, the definition of VesselTransport adopts the first method while HelicopterTransport employs the second. The first method defines an explicit relationship between the VesselTransport and the core description of a vessel outlined in the conveyance section of the integration model. 


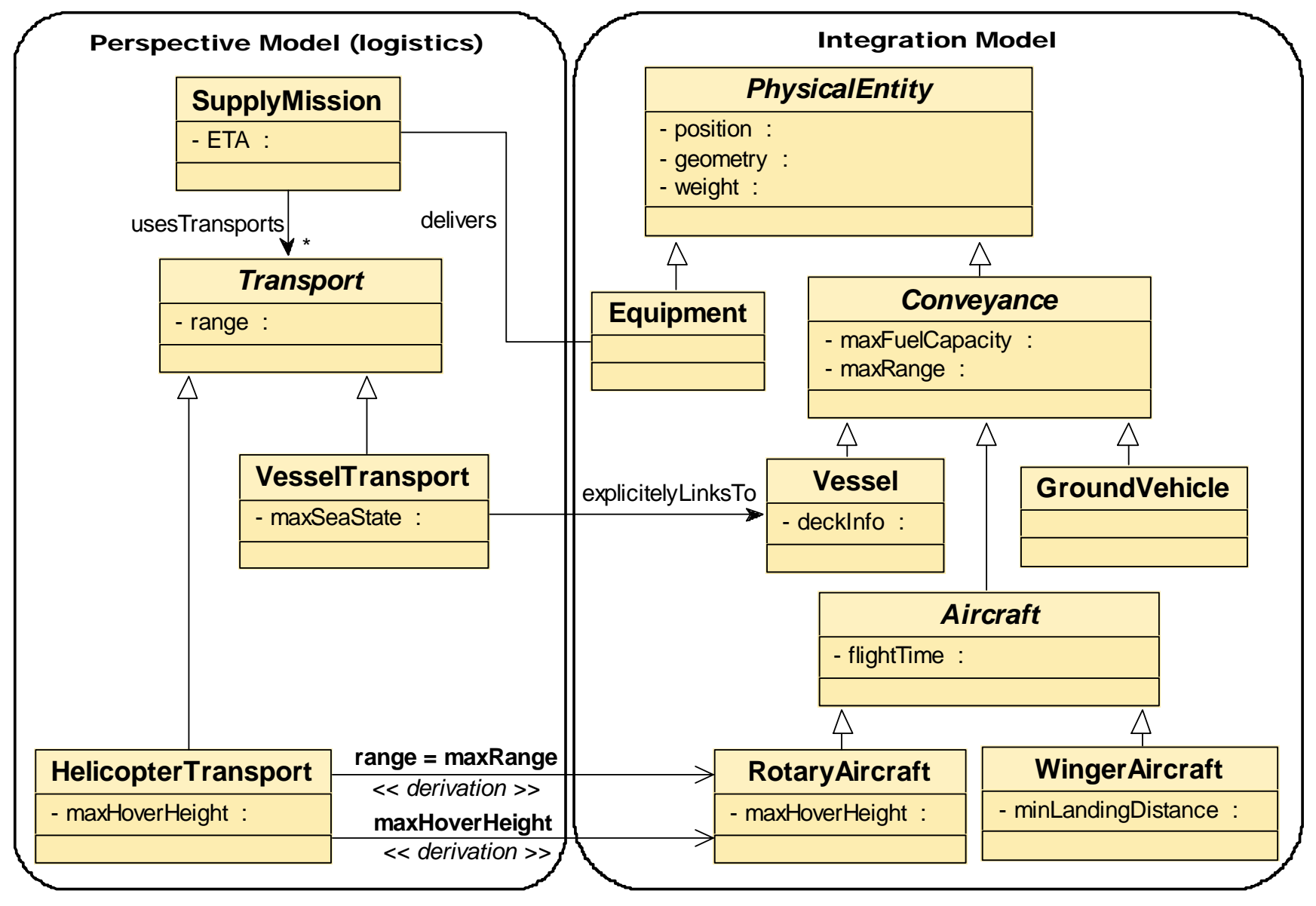

Figure 1 - UML [4] Diagram Illustrating A Logistics Perspective Model Deriving From A Relatively Unbiased Central Integration Model

Utilizing this approach, obtaining the core information relative to the corresponding Vessel from a VesselTransport requires both knowledge of their relationship in addition to a further level of indirection. For reasons of performance and representational precision, both of these requirements may not be desirable.

The second method, illustrated in Figure 1 using HelicopterTransport, overcomes both shortcomings inherent in the first approach. In this case, HelicopterTransport is represented in terms of a façade, or filter of sorts, which transparently connects this biased view to the core RotaryAircraft description housed within the integration model. That is, each attribute of RotaryAircraft desired to be exposed to users of HelicopterTransport is explicitly declared within the façade. For example, since the maximum range of travel is relevant to the definition of a HelicopterTransport the maxRange attribute of RotaryAircraft (inherited from Conveyance) is subsequently exposed in the HelicopterTransport façade. By virtue of being declared as a derived property, any access to such an attribute would be transparently mapped to the corresponding attribute(s) housed within the integration model. In the case of the range attribute of HelicopterTransport, access is transparently directed to the inherited maxRange attribute of RotaryAircraft. Notice also the use of alternative terminology over that used in the integration model (i.e., range vs. maxRange). It should also be noted that the derived nature of a façade attribute is not limited to mapping to a single attribute. Rather, the value of a façade attribute may also be derived through specific behavior, perhaps a calculation based on the values of multiple attributes residing across several integration model objects. In either case, the fact that the value of the façade attribute is derived, and not originating locally, is completely transparent to the façade user.

Another perspective-oriented enhancement to the core integration model illustrated in Figure 1 is the notion of a SupplyMission. Being a fundamental notion of a logistics perspective, a supply mission essentially relates equipment in the form of supply items to the transports by which they will be delivered. Once again, the definition of a logisticsspecific notion (i.e., supply item) is derived from a notion defined in the integration model (i.e., equipment). In this case, an explicit relationship is declared linking SupplyMission to zero or more Equipment items. From the perspective of the logistics system equipment scheduled for delivery is perceived as items to be supplied, the term supplyItems is a more appropriate nomenclature. Such 


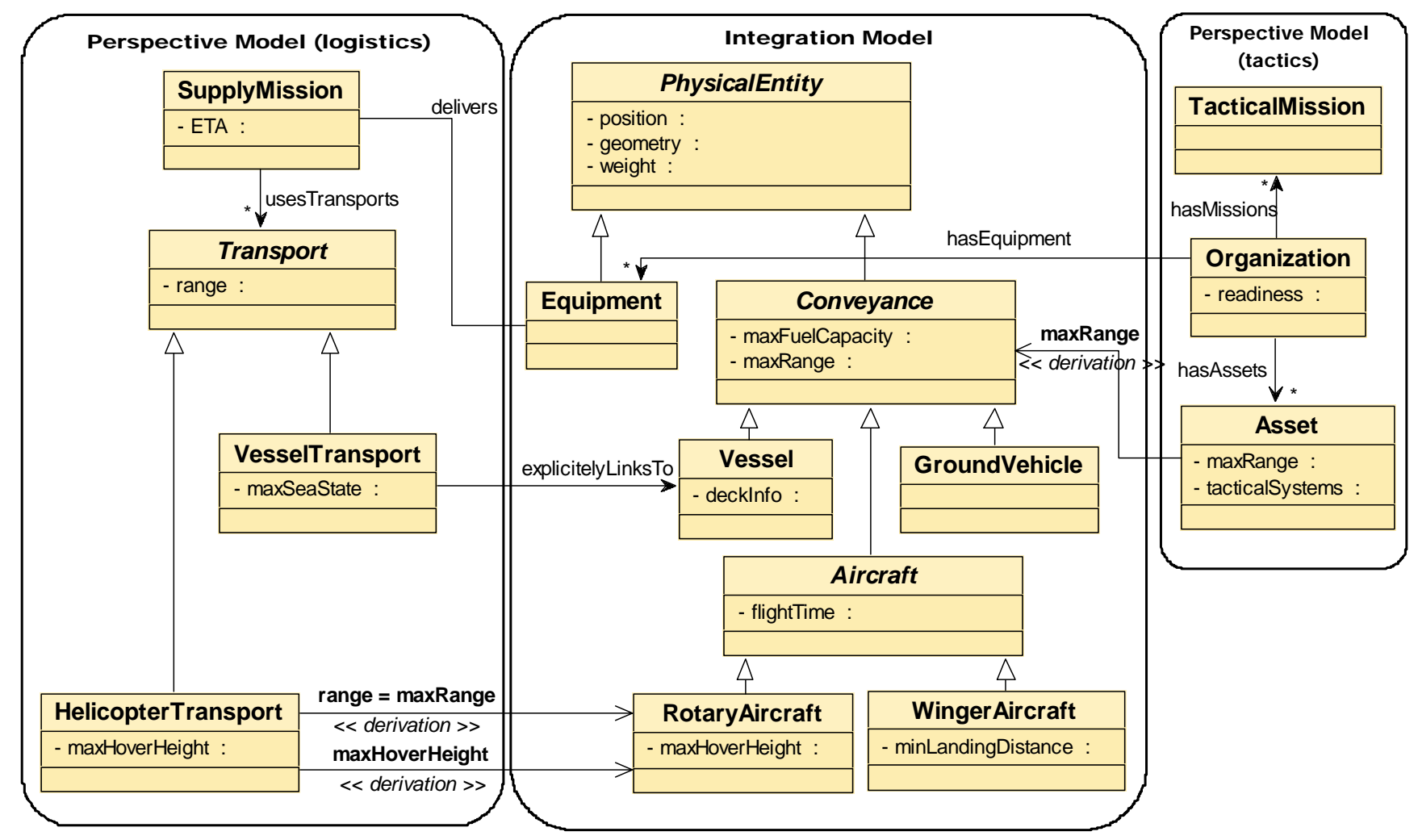

Figure 2 - UML [4] Diagram Illustrating Two Disparate Perspectives Connected Via A Central Integration Model

enhancement to the innate descriptions provided by the integration model demonstrates the ability of a perspective model to essentially overlay new notions (i.e., supply missions) over existing intrinsically-described subject matter (i.e., equipment and conveyances). To further illustrate how multiple, potentially diverse perspectives, can be effectively integrated to support meaningful interoperability, Figure 2 elaborates on the example by introducing an additional perspective on the core subject matter. The additional perspective is that of a particular tactical command and control system. Collaboration between these two perspectives is supported by the common integration model from which many of their notions derive. A conveyance is still a conveyance whether viewed in the context of logistics operations or tactical command and control. Although both users may discuss a conveyance from partially disparate perspectives, both can effectively collaborate about a particular conveyance in terms of their own native, biased perspectives.

\section{An Effective Development Process}

Perspective models can be a powerful means of capturing and exploiting the expressive nature inherent in individuality. However, to arrive at an accepted and effective approach, such a method must be accompanied by a supportive development process. Traditional approaches to domain model development have typically involved a dedicated knowledge engineer, or group of such individuals, whose task it is to produce a well structured representation of the relevant domains of interest. While their efforts may certainly be driven by overall project requirements, they typically direct their focus toward producing an organized domain model. Furthermore, following creation of such a model, component developers design and implement functionality in terms of, or at least in a form that is compatible with, this representation. The problem inherent in this approach is essentially twofold. First, the model development process is not directly influenced by the specific use-cases applied by its actual immediate users. In the end, the primary purpose of the representation sustaining a context-oriented, decision-support environment is to effectively support the software components that are directly using it to both obtain and contribute context. To ensure effective support of these activities, such implicit use-cases should be one-if not the most significant-force that drives model development.

The second pitfall of a conventional approach also deals with the potential disconnect between a subject matter representation and its users. However, in this case the problem manifests itself at a more humanistic level. Critical to the successful application of an often fairly complex representation is the degree to which project team developers embrace, and are able to become familiar with, the various structure and semantics comprising the model. This is especially true in the case of reasoning-based, decision-support systems which tend to operate over complex, highly expressive, and, many times, relationship- 
arduous contexts. To effectively exploit the expressive nature of context-enriched models requires developers to both understand such representation at a semantic level and embrace the manner in which it represents their subject matter interests. Many systems have fallen far short of their potential, sometimes to the point of complete failure, due to a lack of team member understanding and buy-in to the manner in which their domain(s) are represented.

The development process offered in this discussion addresses this disconnect by significantly increasing the involvement of model users with the actual model development activity itself. There are three major benefits to such team member inclusion. First, as component developers research and design their solutions (i.e., software components), they essentially acquire a considerable amount of expertise and knowledge regarding relevant domain(s). Such familiarity goes beyond a fairly deep understanding of the semantics of relevant subject matter to include valuable insight into the precise means by which particular functionality most effectively views, or perceives, such content. It is the identification and subsequent capture of such individualized expression that produces a truly accurate representation. Since the focus is on capturing native perspective and bias, there is no need at this stagein fact it would be potentially polluting - to be concerned about the degree to which these models align with each other. Narrowing the scope of individual perspective model development not only promotes the capture of true individuality, but is also a significantly less complex task than developing a singular, all-encompassing model supporting the entire set of interconnected perspectives. This less complex modeling environment has a direct impact on the amount of expertise and experience required for effectively developing these personalized perspective models. While good modeling practices are still quite important in this process, they can be applied within considerably less complex environments by individuals who may not have the modeling depth of an experienced knowledge engineer. Further, familiarity with model structure and subsequent semantics undoubtedly leads to a significantly stronger bond between component developers and the subject matter representation over which their components operate.

The second component to the integration architecture described above is the integration model that effectively ties related perspective models together. Development of this model is a considerably more complex task than development of the perspective models themselves. Development of the integration model involves the analysis of each perspective model with an eye for both identifying and abstracting subject matter existing across the multitude of user perspectives. Considering the complexities involved in this analysis in addition to the demand for being both knowledgeable and comfortable with applying various intricate analysis patterns, this activity typically requires a highly experienced expert modeler. This activity, for example, might become the main area of focus for the expert knowledge engineer(s) who have traditionally been responsible for the entire modeling activity. Many of the same concerns critical to successfully developing a traditional model (e.g., model integrity, extensibility, clarity, accuracy, etc.) are quite applicable to the development of integration models as well.

The final component to building the integration model is to describe the relationships between the various perspective models and the central integration model from which they derive. Recall that such mappings can be described in terms of a formalized expression language. Coupled with some type of code-generation facility capable of managing implementation concerns, these mappings can be designed, communicated, and maintained primarily at the modeling level. Similar to development of the actual integration model itself, development of these mappings will likely also require the skills of an experienced knowledge engineer.

\section{CONCLUSION}

To obtain truly accurate, expressive representation, individual perspective must be specifically captured based on the use-cases of its immediate user(s). Interoperability within a diverse, perspective-enriched environment must support meaningful interaction between users that preserves individualized perspective. Applying perspective models, interconnected via a unifying integration model, effectively supports these two objectives. In addition, employing a development process where perspective model development is directly driven by the needs of the immediate users leads to a more precise and expressive representation while significantly improving the representation's effectiveness through increased user familiarity and imperative model acceptance.

\section{REFERENCES}

[1] Cagle, K., M. Corning, J. Diamond, T. Duynstee, O. Gudmundsson, M. Mason, J. Pinnock, P. Spencer, J. Tang, A. Watt, J. Jirat, P. Tchistopolskii, and J. Tennison, "Professional XSL", Wrox Press Ltd,. Birmingham, UK., 2001

[2] Daconta M., L. Obrst and K. Smith, "The Semantic Web: A Guide to the Future of XML, Web Services, and Knowledge Management”, Wiley, Indianapolis, IN., 2003

[3] Fowler, M., “Analysis Patterns: Reusable Object Models”, Addison-Wesley, Reading, Massachusetts, 1997.

[4] Fowler, M., "UML Distilled: Applying the Standard Object Modeling Language”, Addison-Wesley, Reading, Massachusetts, 1997.

[5] Fowler M., D. Rice, M. Foemmel, E. Hieatt, R. Mee, and R. Stafford, "Patterns of Enterprise Application 
Architecture”, Addison-Wesley, Reading, Massachusetts, 2003

[6] Friedman-Hill, E., “JESS In Action”, Manning Publications Co., Greenwich, CT, 2003

[7] Garshol L. and G. Moore (eds.), "The XML Topic Maps (XTM) Syntax”, JTC1/SC34:ISO 13250, July 22, 2002, (www.y12.doe.gov/sgml/sc34/document/0328.htm)

[8] Giarratano J. and Riley G., "Expert Systems: Principles and Programming”, $2^{\text {nd }}$ Edition, PWS Publishing Company, Boston, MA.

[9] Hunter D., C. Cagle, D. Gibbons, N. Ozu, J. Pinnock, and P. Spencer, "Beginning XML", Wrox Press Ltd., Birmingham, UK., 2000

[10] Karsai G., "Design Tool Integration: An Exercise in Semantic Interoperability”, Proceedings of the IEEE Engineering of Computer Based Systems, Edinburgh, UK, March, 2000

[11] NASA, “CLIPS 6.0 Reference Manual”, Software Technologies Branch, Lyndon B Space Center, Houston, Texas, 1992

[12] Pohl J., "Information-Centric Decision-Support Systems: A Blueprint for Interoperability”, Office of Naval Research (ONR) Workshop hosted by the CAD Research Center in Quantico, VA, June 5-7, 2001

[13] Pohl J, A Chapman, K Pohl, J Primrose and A Wozniak, "Decision-Support Systems: Notions, Prototypes, and InUse Applications”, Technical Report, CADRU-11-97, CAD Research Center, Design Institute, College of Architecture and Environmental Design, Cal Poly, San Luis Obispo, CA, January, 1997

\section{AUTHOR BIOGRAPHY}

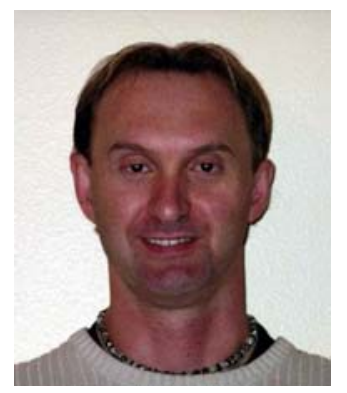

Kym J. Pohl is a senior software engineer and a co-director of CDM Technologies, Inc. in San Luis Obispo. His current focus is agentbased, collaborative decisionsupport systems with particular interest in representation and collaboration architectures. Following an undergraduate degree in Computer Science he earned Master's degrees in both Computer Science and Architecture. Over the past 18 years he has provided technical leadership in the design and development of a number of context-oriented, decision-support systems for the US Department of Defense, including the Integrated Marine Multi-Agent Command and Control System (IMMACCS) for tactical command and control and the
SEAWAY system for the coordination of logistical sea-based sustainment operations. 